\title{
Improved taxonomic assignment of human intestinal 16S rRNA sequences by a dedicated reference database
}

\author{
Jarmo Ritari ${ }^{1 *}$, Jarkko Salojärvi ${ }^{1}$, Leo Lahti ${ }^{1,2}$ and Willem M. de Vos ${ }^{1,2,3}$
}

\begin{abstract}
Background: Current sequencing technology enables taxonomic profiling of microbial ecosystems at high resolution and depth by using the $16 \mathrm{~S}$ rRNA gene as a phylogenetic marker. Taxonomic assignation of newly acquired data is based on sequence comparisons with comprehensive reference databases to find consensus taxonomy for representative sequences. Nevertheless, even with well-characterised ecosystems like the human intestinal microbiota it is challenging to assign genus and species level taxonomy to $16 \mathrm{~S}$ rRNA amplicon reads. A part of the explanation may lie in the sheer size of the search space where competition from a multitude of highly similar sequences may not allow reliable assignation at low taxonomic levels. However, when studying a particular environment such as the human intestine, it can be argued that a reference database comprising only sequences that are native to the environment would be sufficient, effectively reducing the search space.

Results: We constructed a 16S rRNA gene database based on high-quality sequences specific for human intestinal microbiota, resulting in curated data set consisting of 2473 unique prokaryotic species-like groups and their taxonomic lineages, and compared its performance against the Greengenes and Silva databases. The results showed that regardless of used assignment algorithm, our database improved taxonomic assignation of 165 rRNA sequencing data by enabling significantly higher species and genus level assignation rate while preserving taxonomic diversity and demanding less computational resources.
\end{abstract}

Conclusion: The curated human intestinal 16S rRNA gene taxonomic database of about 2500 species-like groups described here provides a practical solution for significantly improved taxonomic assignment for phylogenetic studies of the human intestinal microbiota.

Keywords: Next-generation sequencing, 16S, Ribosomal RNA, Human intestinal microbiota, Bacteria, Archaea, Taxonomy

\section{Background}

As the most genetically diverse and functionally complex microbial ecosystem of the human body the intestinal microbiota has become one of the major areas of interest in microbial ecology [1]. In particular, efforts have been undertaken to understand how individual composition and variation of the microbiota together with host genetic and environmental factors influence human health [2,3]. Over the past decade it has become evident that the microbiota exerts various beneficial effects to the host

\footnotetext{
* Correspondence: jarmo.ritari@gmail.com

'Department of Veterinary Biosciences, University of Helsinki, Helsinki, Finland Full list of author information is available at the end of the article
}

physiology during the development and in adulthood, notably through immunity and nutrition $[4,5]$, and deviations from a balanced microbial composition are related to systemic problems, such as diabetes, obesity and allergy [6-8]. Progress in molecular analysis of the microbiota has been made possible largely by the advance of next-generation sequencing technology, which has allowed studying the composition and dynamics of microbial communities with unforeseen scale and resolution $[9,10]$.

The bacterial and archaeal $16 \mathrm{~S}$ small subunit ribosomal RNA (16S rRNA) gene has been established as the most widely used phylogenetic marker due to its conserved and variable regions and universal presence in prokaryotes. By sequencing the pool of $16 \mathrm{~S}$ rRNA genes, 
community composition can be investigated in a comprehensive and rapid manner by high-throughput sequencing platforms harbouring the capacity for millions of reads per single run $[11,12]$. As a result of increasing read length, sample multiplexing capability and reducing costs, $16 \mathrm{~S}$ sequence data is being accumulated from various microbial ecosystems, and vast reference databases like Silva [13, 14], Greengenes (GG) [15] and RDP [16] have been built to enable phylogenetic analysis of highthroughput data.

While being highly successful at gathering data, the high-throughput technologies also present challenges for data analysis by requiring sophisticated computational methods not only in correcting technical artifacts but also for organizing the output and extraction of biologically meaningful features. A crucial step in deciphering $16 \mathrm{~S}$ rRNA reads data is the taxonomic annotation of the discovered sequences. This holds true especially because current sequencing technologies typically cover only a part of the 16S rRNA gene, the large number of reference sequences and limited resolution at genus and species levels $[17,18]$. Taxonomic annotations have been shown to depend on several factors, including sequence length, target region of the $16 \mathrm{~S}$ gene, OTU classification method and assignment algorithm. Although many comparative studies have addressed these technical factors [19-22], the effect of the reference database on the accuracy of taxonomic assignment remains less well known. The standard approach has been to use as comprehensive a database as possible to minimize the number of unclassified sequences [23]. However, increasing database size also makes it potentially more difficult to assign taxonomy at genus and species levels as the likelihood of ambiguous assignment increases due to larger number of competing sequences in the search space. On the other hand, better taxonomic resolution would be valuable in profiling the human gut microbiota because different species and genera can associate with different conditions and outcomes [24]. Furthermore, the $16 \mathrm{~S}$ rRNA gene has been shown to have considerably higher ambiguous assignment rate at lower taxonomic levels compared with other taxonomic marker genes [18], making its use somewhat problematic despite extensive reference data sets.

We hypothesized that by reducing the size of the reference database to encompass only the sequences innate to the environment under study would lead to improved taxonomic assignations at lower taxonomic levels due to less competition among targets. In this respect, the human intestinal microbiota presents an advantageous model system because it is already well characterized by sequencing $[25,26]$ while genus and species level taxonomic assignment of new sequencing data remains challenging [17]. Moreover, a curated set of over 1000 cultured bacterial and archaeal species from the human intestinal ecosystem has recently been reported [27]. To this end, we constructed a custom human intestinal 16S rRNA database, termed HITdb, including all currently known cultivable gastrointestinal prokaryotes as well as operational taxonomic units (OTUs) generated from high-quality $16 \mathrm{~S}$ rRNA sequences originating from human intestinal tract. Here, we have evaluated the taxonomic assignment performance of the custom database by comparing it with the current standard, the Greengenes database, and demonstrate that the custom database improves taxonomic assignation of human intestinal $16 \mathrm{~S}$ high-throughput reads. The 2473 species-like 16S rRNA sequences present in the HITdb also provide a minimum estimate for the number of species present in the human intestinal ecosystem.

\section{Results and discussion}

To construct the human intestinal microbiota 16S rRNA database (HITdb), we extracted a subset from Greengenes and Silva databases by using a set of over 73,000 NCBI GenBank 16S rRNA sequences annotated as originating from the human gastrointestinal tract. Pulling down the human intestinal subset from Silva and Greengenes by matching the GenBank sequences at $97 \%$ global similarity (used as an OTU definition throughout this work) resulted in over 650,000 sequences, which were further filtered from potential chimeras and shorter than $1.3 \mathrm{~kb}$ sequences to the final number of 531,712 sequences (Fig. 1). Clustering the sequences using presently known cultivable species [27] as reference resulted in altogether 1482 bacterial and 27 archaeal de novo OTUs. In total the database contained 2473 species-like clusters (Fig. 1). By including only curated and near full-length sequences and requiring at least two sequences per cluster (i.e. nonsingletons) we aimed to minimize the possibility of generating spurious OTUs, which are prone to occur with short or chimeric sequences $[28,29]$. Each de novo OTU represents at least $3 \%$ sequence identity difference to other OTUs and known species. Although the $3 \%$ is only an arbitrary threshold and differences in genetic distances between taxonomic groups vary so that OTUs may not be monophyletic [30,31], it is commonly accepted as an approximate species assignment in $16 \mathrm{~S}$ analysis $[32,33]$. Defining the OTUs by sequence identity is to some extent facilitated by using near full-length $16 \mathrm{~S}$ sequences, which provide more robustness in contrast to smaller fragments of the rRNA gene where the application of the $97 \%$ rule would become more problematic. Although other clustering methods exist that show improvement relative to a strict identity cutoff based OTU definition [30, 34], they tend to be expensive in terms of required computational resources and thus challenging for processing large (i.e. > $10^{5}$ sequences) datasets like in this study. For example, the heuristic OTU clustering algorithm Uclust applied here to 


\section{NCBI Genbank human} intestinal $16 \mathrm{~S}$

73,000

\section{Extract from Silva and Greengenes $16 \mathrm{~S}$ sequences at $97 \%$ identity} 650,000

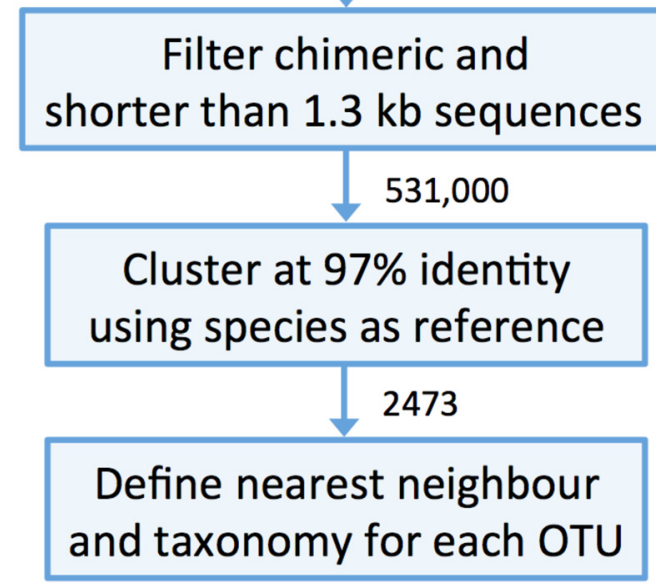

Fig. 1 Main steps in the construction of the human intestinal tract $16 \mathrm{~S}$ taxonomic database (HITdb). Human intestinal specific sequences were pulled down from the Greengenes and Silva databases using Genbank sequences. Obtained sequence data were clustered at $97 \%$ identity by using cultivable human intestinal species as a reference. A cultivable nearest neighbour was determined for each OTU. The taxonomic lineages were determined based cultivable species taxonomy, Greengenes and manual curating

construct HITdb is slightly less robust than the UPGMA method [31] but efficient with large datasets.

Finally, the HITdb sequences were taxonomically assigned based on the cultivated species' taxonomy, Greengenes and manual curating. A nearest neighbour cultivable species was determined for each OTU to facilitate the interpretation of the OTUs. Phylogenetic trees constructed from bacterial and archaeal sequences (Additional file 1) were found to correspond with the nearest neighbour information.

In order to evaluate how comprehensively the HITdb represents taxonomic diversity we performed a computational rarefaction analysis based on the sequence data used for constructing the HITdb (see Methods). The obtained rarefaction curve shows that the number of $97 \%$ OTUs is not quite saturated at current sequence data (Additional file 2), which would indicate that the full species-level diversity is not fully covered. On the other hand, actual rarefaction by sampling random subsets from the sequence data, defining OTUs for each sampled subset and calculating the number of known unique species and genera represented by the OTU clusters showed that the numbers were not significantly lower in samples constituting about $80 \%$ of the original sequence data (Additional file 3), suggesting that the data is close to reaching saturation. Altogether, these results suggest that the HITdb is able to capture the diversity of known taxa quite well, because in all tests with actual rarefaction the observed numbers would be expected to drop significantly if the sampling depth was a limiting factor. However, the sequence coverage in clusters representing known species was typically higher than in OTUs (data not shown), which could explain why saturation is seen with known species in actual rarefaction, but not when all OTUs are considered in computational rarefaction. Since most of the $16 \mathrm{~S}$ sequences are assigned to clusters of known species, it also implies that if some species-like groups were missing from HITdb, they would be increasingly rare and therefore probably not highly relevant.

The number of entries in the curated HITdb, viz. a total of 2473, can be seen as the present estimate for the minimal number of species expected to be present in the human intestinal tract. Since according to the rarefaction analysis the number of OTUs is probably not quite saturated yet, the number may still increase with new data. However, there may be only a limited number of new OTUs emerging, similar to the situation with metagenomic data that shows only a limited increase to the known information pool with the addition of new metagenome sequences [35]. In any case, an earlier estimate of about 1800 human intestinal species remaining uncultured [36] is consistent with HITdb because the number of cultured species has increased since then [27], leaving about 1500 species still uncultured. This is an important estimate to keep in mind when designing strategies to culture the not-yet cultured species from the human intestinal tract.

To assess the performance of HITdb in taxonomic assignment we compared it with Greengenes by analysing synthetic data constructed from the sequences of known human gut resident species (Additional file 4). Since the taxonomic position of the synthetic reads is known, it was possible to evaluate the effect of the used database without potential confounding factors such as sequencing technology or unknown sequence content that might favour one database over another. We first determined the relationship between assigned and unassigned taxa at different taxonomic levels for the two tested databases (Fig. 2a). The results show that the relative numbers of missing genus-level assignations was below $80 \%$ with Greengenes, irrespective of used $16 \mathrm{~S}$ region or assignment algorithm, while with HITdb there were practically no missing assignments observed. At the level of species the difference was even more pronounced since with Greengenes only 


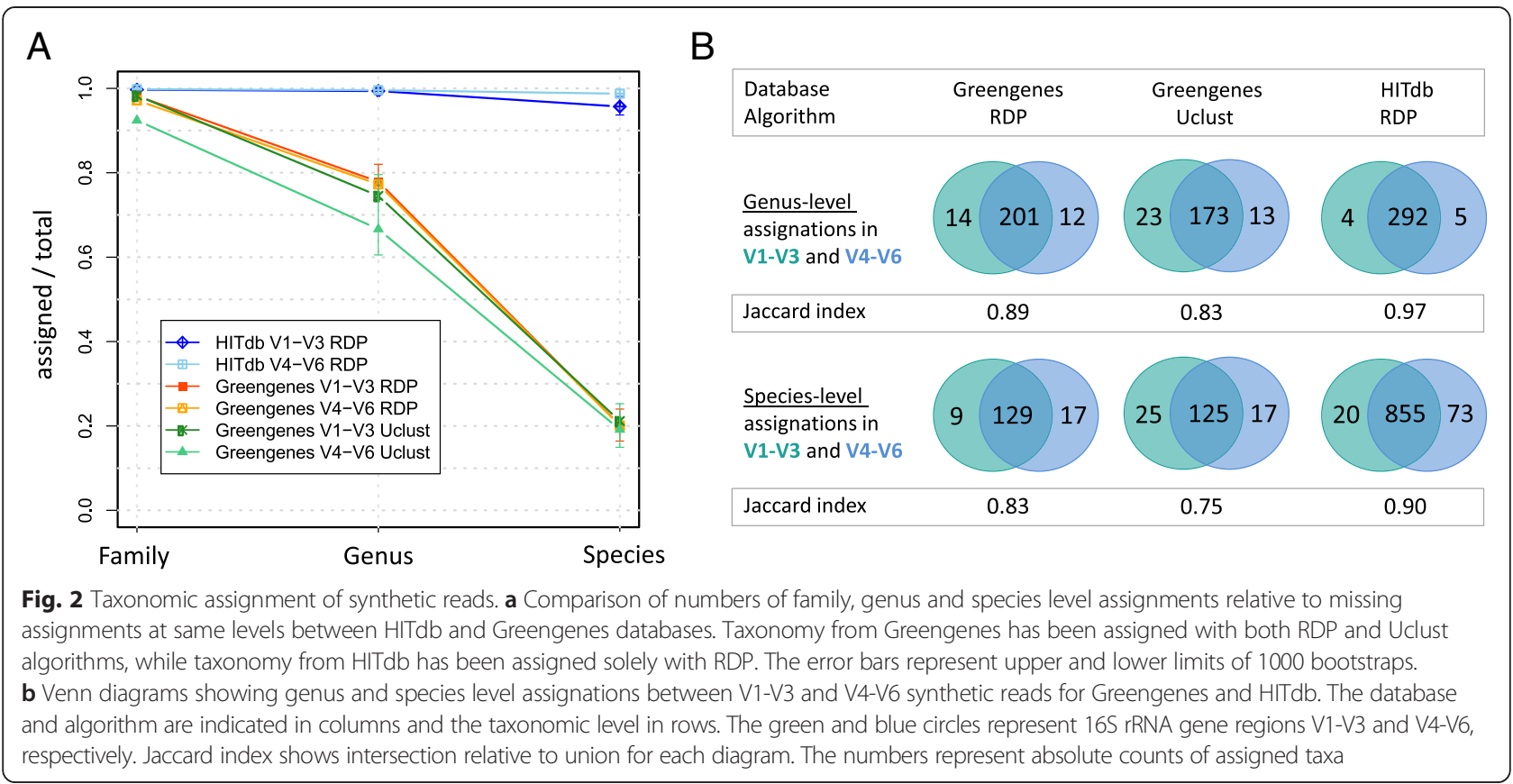

about $20 \%$ of synthetic reads could be assigned to specieslevel, while with HITdb over $90 \%$ assignation rate was achieved. Similar results were obtained by using Mothur as the assigner algorithm and Silva as the reference database, although with Silva the assignment rate at all tested levels was extremely low (Additional file 5) and thus Silva was not included in further comparative analyses. It should be notified that although HITdb contains the synthetic read parent sequences, the same should also be true for Greengenes because only known species were included in the analysis, so potentially missing taxa cannot explain the observed difference. The assigner algorithm is also not likely to be explanatory for this result because three different approaches were compared; naïve Bayesian (RDP) [37] and Mothur [34], and sequence similarity majority vote based Uclust (as part of the Qiime pipeline).

Secondly, we compared the agreement of taxonomic assignations between two different regions of the $16 \mathrm{~S}$ rRNA gene, V1-V3 and V4-V6, by using GG and HITdb. At both genus and species levels the number of shared assignments relative to all assignments was better with HITdb, as shown by the Jaccard index and absolute numbers of shared assignments (Fig. 2b). Moreover, accuracy estimation of synthetic read assignments between Greengenes and HITdb showed that HITdb performs better in terms of absolute and relative correct assignments (Fig. 3). These results further suggest that the database itself is a major determinant in genus and species level assignment performance. This is an important observation, as one of the limitations in current intestinal microbiota analysis is the assignment at the species level and its subsequent interpretation.
To further evaluate the performance of HITdb we analysed high-throughput $16 \mathrm{~S}$ amplicon reads data generated from two sets of fecal samples by pyrosequencing and paired-end Illumina technologies (Fig. 4). We found that the number of species and genus level assignations was higher in both absolute and relative terms with HITdb in contrast to Greengenes. Since Greengenes does not contain identifiable OTUs (but just missing taxonomic information for unknown groups), we removed the OTUs from HITdb assignation results and included only known species (indicated by gray boxplots in Fig. 4). Also in these comparisons the number of assigned taxa was significantly higher with HITdb, further confirming the observation that the database search space size itself is likely to be an important factor in taxonomic assignment. To rule out other biases caused by different numbers of non-assigned reads, we compared the numbers of reads not assigned to any phylum in HITdb and Greengenes. The numbers of non-assigned reads in HITdb assignations were at least as low as in Greengenes, amounting to approximately $0.1 \%$ of total (Additional file 6). This indicates that the HITdb enables comprehensive assignment in our set of test samples in a $16 \mathrm{~S}$ region and sequencing technology independent manner, and in general suggests that HITdb does not limit taxonomic assignment despite being much smaller in content than Greengenes.

To confirm the results on biological data with an independent data set and methodology, we performed comparative tests using publicly available Human Microbiome Project (HMP) data. HITdb performance was first compared with Greengenes in 192 fecal $16 \mathrm{~S}$ sequencing samples (Additional file 7). The results indicate that HITdb 


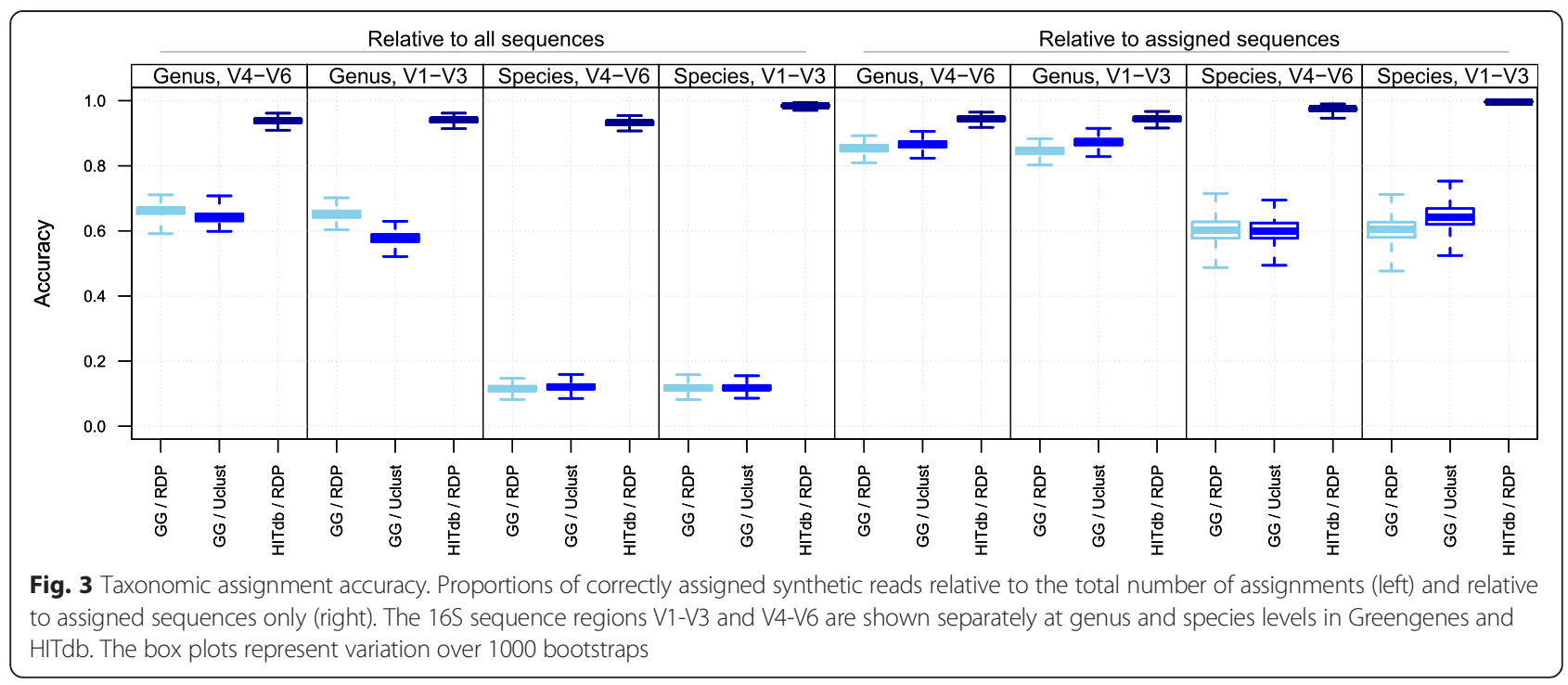

was able to detect a higher number of genera and species than Greengenes from these data as well (Fig. 5a), being consistent with results from other data sets analysed in similar way. Furthermore, we compared taxonomic profiles from our $16 \mathrm{~S}$ analysis with shotgun metagenome taxonomic profiling available in HMP. The numbers of genera and species shared with the metagenomic profiles were again higher in HITdb than in Greengenes (Fig. 5b), suggesting better accuracy for HITdb. All in all, the comparative test results suggest that HITdb outperforms Greengenes in quantity and quality independently of data source.

We also tested how well the relative numbers of taxa correlated between HITdb and Greengenes (Fig. 6). When

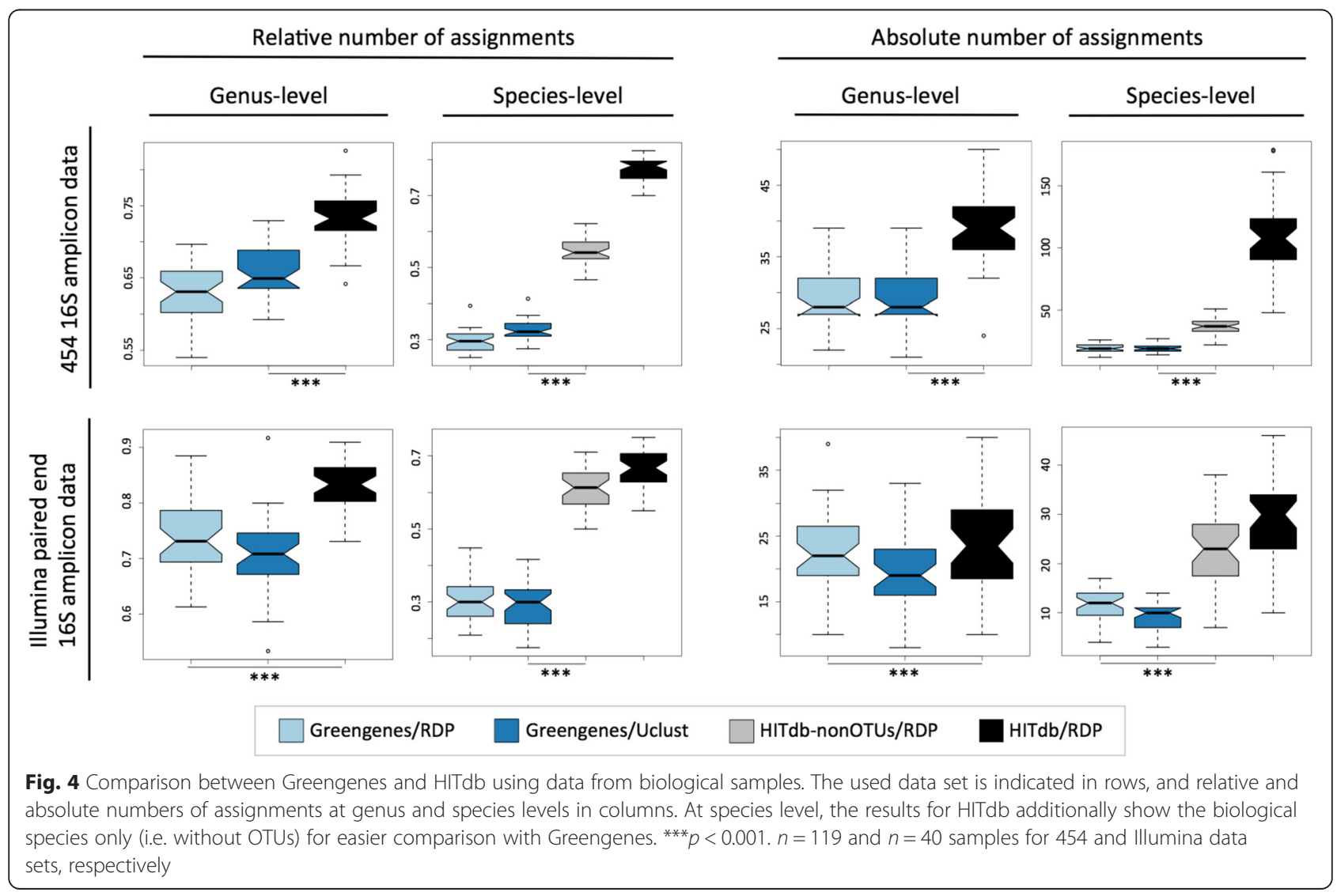



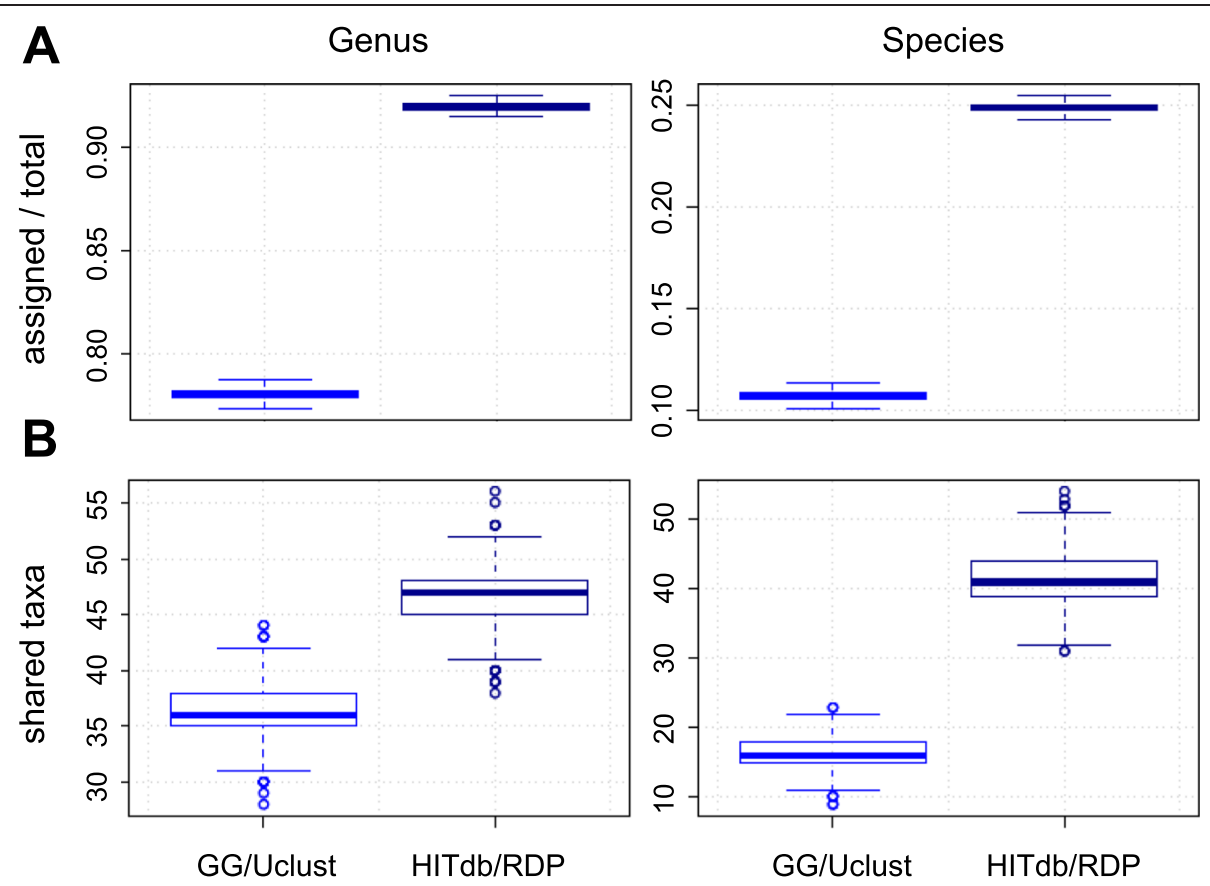

Fig. 5 Analysis of Human Microbiome Project data. a Numbers of genera and species found by Greengenes and HITdb in HMP 16S samples ( $n=192$ ). b Numbers of shared genera and species between HMP shotgun metagenomics profiling and $16 \mathrm{~S}$ analysis of the same HMP samples by Greengenes and HITdb $(n=23)$. The boxplots represent variation over 1000 bootstraps

considering genera averaged over all samples, or samples averaged over all genera, the Pearson correlation coefficients were over 0.95 . Moreover, when considering all data points (i.e. single genus in single sample), the correlation coefficient was over 0.8 , showing that HITdb largely agrees with Greengenes in a quantitative manner. Differences are also quite symmetrical over the diagonal meaning that HITdb doesn't have tendency to systematically over- or underrepresent taxa abundances relative to Greengenes.

Computational resources required by taxonomic annotation depend on the reference database, assignment algorithm and the number of sequences to be assigned. We found that HITdb with RDP classifier is both faster and takes less memory than Greengenes using Uclust or RDP classifiers (Additional file 8). Although Uclust is very fast, it is quite memory intensive, while RDP (and Mothur) consume less memory but are slower. Since HITdb performs faster and with less memory than either algorithm with Greengenes, it may be expected to scale well for increasingly large datasets for future needs.

\section{Conclusions}

Profiling the composition of intestinal microbiota relies on accurate taxonomic annotation of sequencing reads. However, large reference databases may not be able to provide optimal species- and genus-level resolution due to increasing competition in the search space. To improve low-level resolution without abandoning comprehensiveness in taxonomic assignation we propose using a dedicated reference database specific to the ecosystem under study. By constructing a human intestinal $16 \mathrm{~S}$ database and comparing its performance with Greengenes we found that the dedicated reference database improves the assignment rate at genus and species level, suggesting that large search space may be a limiting factor in low-level taxonomic assignment. Our study provides a practical solution with considerable performance improvements, is readily applicable in human gut microbiota profiling studies and paves the way for developing similar focused databases for other model systems.

\section{Methods}

\section{HITdb construction}

To create a reference data set the $16 \mathrm{~S}$ sequences of cultivable bacterial and archaeal human intestinal resident species [27] were obtained from NCBI Genbank. In addition, a Cyanobacteria related Melainabacterium species from curated metagenome [38], a cultivable representative of phylum TM7 from human oral cavity [39], Intestinimonas butyriproducens and Methanomassiliicoccus intestinalis [40] were included to the reference set of known species.

To obtain a comprehensive set of near full-length $16 \mathrm{~S}$ sequences originating from human intestinal microbiota a search was performed against the NCBI Genbank nucleotide database using the command (("Homo sapiens"[Organism] OR human[All Fields]) AND (intestinal[All Fields] 


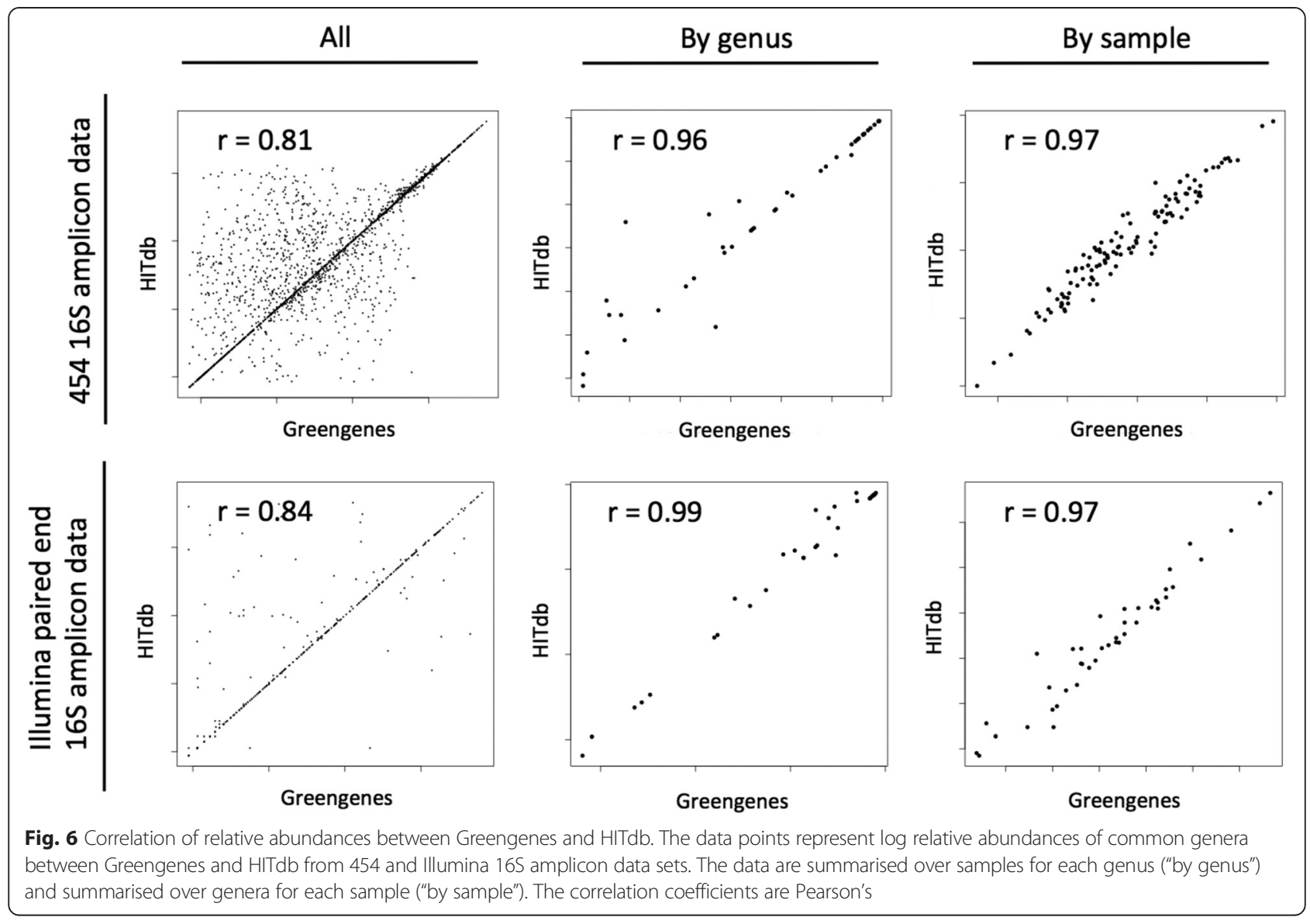

OR gut[All Fields]) AND 16S[All Fields]) AND ("bacteria"[porgn] OR "archaea"[porgn]) AND 1000:2000[SLEN]. The extracted sequences were matched against the Greengenes 13_5 [15] and Silva [13, 14] (SSURef_NR99_115_tax_silva_trunc) $16 \mathrm{~S}$ databases at $97 \%$ identity using Usearch v. 7.0.1001 command usearch_global. The matched sequences were extracted from both databases and subjected to chimeric sequence removal by UCHIME v. 7.0.1001 (command uchime_ref; default parameters) using the $16 \mathrm{~S}$ reference database available at http://drive5.com/uchime/gold.fa [41]. The non-chimeric sequences were length filtered to exclude sequences shorter than $1.3 \mathrm{~kb}$. The filtered sequence data was then clustered to OTUs using the cultivable species' sequences as a reference but allowing non-matching sequences to cluster de novo. At minimum two sequences were required for each de novo OTU. The OTU clustering was performed at $97 \%$ identity threshold in Qiime v. 1.8.0 [42] using the command pick_open_reference_otus.py with parameters suppress_taxonomy_assignment, min_otu_size $=2$, prefilter_percent_id $=$ 0.0 , percent_subsample $=0.1$ and suppress_align_and_tree. Next, the representative sequences of OTU clusters were matched back to the reference species' sequences using Usearch v. 7.0.1001 command usearch_global with parameters id $=0.5$ and maxhits $=1$. OTUs having a match over $97 \%$ similar to any of the cultivable species were removed (i.e. collapsed with the corresponding species). Furthermore, for each OTU, the nearest cultivable species was determined from the sequence match results. The final sequence content of HITdb consisted of representative sequences of the processed de novo OTUs and cultivable species.

HITdb OTU representative sequences were assigned taxonomy from the taxonomy of known species and Greengenes by using RDP classifier. In cases where HITdb was able to give a lower level assignation than Greengenes, and where all taxonomic levels were in agreement between HITdb and Greengenes, the OTU was assigned the taxonomy given by the known species. The lineages of species and OTUs were manually checked for consistency, and to adapt them to current naming convention as well as possible.

\section{Phylogeny}

The HITdb bacterial and archaeal sequences were separately aligned using Muscle v. 3.8.31 with default settings [43]. The alignments were filtered in Qiime v. 1.8.0 using command filter_alignment.py with parameter 
suppress_lane_mask_filter. Newick formatted phylogenetic trees were built from the filtered alignments using FastTree [44]. The trees were visualized with FigTree v. 1.4.0 (http://tree.bio.ed.ac.uk/software/figtree/).

\section{Synthetic reads}

The $16 \mathrm{~S}$ sequences of 953 cultivable human intestinal bacterial species were aligned to 518R [45] and 338R [46] primer sequences allowing 2 or 3 mismatches, respectively, in order to extract V1-V3 and V4-V6 gene regions from the sequences. For V1-V3, target sequence from its start until the end of 518R alignment position was extracted. For V4-V6, sequence from 338R alignment start position until $500 \mathrm{bp}$ downstream of the target was extracted. The synthetic read sequences are given in Additional file 4.

\section{Taxonomic assignments}

Both biological and synthetic $16 \mathrm{~S}$ reads were taxonomically assigned using in-built functions of Qiime v. 1.8.0 (assign_taxonomy.py, make_otu_table.py, summarize_taxa_through_plots.py) [42] with default parameters except for reference database where in addition to Greengenes 13_5 [15] also HITdb and Silva were used, and assignment algorithm where RDP [37] and Mothur [34] were used along with the default Uclust.

\section{Biological samples and $16 \mathrm{~S}$ amplicon sequencing}

Two sets of fecal samples obtained from children were sequenced for evaluating the HITdb performance with real data. Sample collection and DNA extraction were performed as described before [48, 49]. For data set 1 (119 samples) [47], PCR amplicons from bacterial 16S rRNA gene region V4-V6 were generated with forward (5'-AYT GGGYDTAAAGNG-3') and reverse (5'-TGCTGCCTCCC GTAGGAGT-3') primers. For data set 2 (40 samples) [48], amplicons from V1-V3 region were generated with forward (5'-AGAGTTTGATCMTGGCTCAG-3') and reverse (5'-G TATTACCGCGGCTGCTG-3') primers. The PCR primers contained 18-mer overhangs added to the 5' ends [49]. Replicate PCR products were pooled and purified with Agencourt AMPure XP magnetic beads (Agencourt Bioscience) and subjected to a second PCR round with barcoded forward primers and a reverse primer, both of which attached to the respective 18-mer overhang sequences from the primers of the first PCR amplification. Phusion polymerase (Thermo Fisher Scientific/Finnzymes) with HF buffer and $2.5 \%$ DMSO were used. Cycling conditions for both PCR reactions consisted of an initial denaturation at $98{ }^{\circ} \mathrm{C}$ for $30 \mathrm{~s}$, followed by 15 cycles at $98{ }^{\circ} \mathrm{C}$ for $10 \mathrm{~s}, 65^{\circ}$ $\mathrm{C}$ for $30 \mathrm{~s}$, and $72{ }^{\circ} \mathrm{C}$ for $10 \mathrm{~s}$, and then a final extension for $5 \mathrm{~min}$. Between 3.6 and $60 \mathrm{ng}$ of template DNA were used in the initial reaction. DNA concentration and quality were measured with Qubit (Invitrogen) and Bioanalyzer
2100 (Agilent). Sample set 1 was sequenced on 454 FLX Titanium instrument and set 2 in paired-end mode (R1 = $326 \mathrm{bp}, \mathrm{R} 2=286 \mathrm{bp}$ ) on Illumina MiSeq instrument with standard library preparation protocol. Sequencing was carried out at the DNA sequencing and genomics laboratory, Institute of Biotechnology, University of Helsinki, Finland.

\section{Pre-processing of $16 \mathrm{~S}$ amplicon sequencing data}

The raw pyrosequencing reads were subjected to referencebased chimera filtering using UCHIME v. 7.0.1001 [41] (command uchime_ref; default parameters) with $16 \mathrm{~S}$ reference database available at http://drive5.com/uchime/gold.fa. The non-chimeric reads were length filtered to exclude reads shorter than $500 \mathrm{nt}$. Thereafter the read numbers were rarefied by randomly sampling the lowest common read number (4246) from each sample using the Biostrings library [50] in R v. 3.1.1 [51]. The reads from paired-end Illumina MiSeq sequencing data set were treated in a similar manner, except for merging of read pairs which was performed using Usearch v. 7.0.1001 command fastq_mergepairs [52] with parameters fastq_truncqual $=4$, $\operatorname{minhsp}=9$, fastq_minovlen $=10$, fastq_maxdiffs $=3$ and fastq_minmergelen $=440$. The quality filtering of the merged read pairs was done with Usearch fastq_filter with parameters fastq_truncqual $=10$ and fastq_maxee $=0.75$. The merged and filtered Illumina reads were rarefied to 13,303 reads per sample.

\section{HMP data analysis}

16S data of 192 Human Microbiome Project fecal samples were obtained from Sequence Read Archive (http:// sra.dnanexus.com/). The SRA sample ID codes are given in Additional file 7. The data were preprocessed as described above, except for using minimum sequence length cutoff of $400 \mathrm{nt}$ and rarefaction cutoff of 4000 reads. The data were analysed in Qiime v. 1.9 with default parameter settings. The data were taxonomically assigned by Uclust and Greengenes v.13_8, and by HITdb and RDP classifier in Qiime.

Metagenomic, taxonomically profiled data from HMP were obtained from HMSMCP - Shotgun MetaPHIAn Community Profiling (http://www.hmpdacc.org/HMSM $\mathrm{CP} /$ ). The samples with the same SRS codes as in $16 \mathrm{~S}$ HMP data were selected for taxonomic comparison. For HITdb, OTUs were excluded from comparative analysis at species level to make comparisons equal.

\section{Statistical methods}

In order to estimate completeness of sequence data used to define the HITdb OTUs, sampling from multinomial distribution was performed. The number of sequences binned to each species-like cluster constituted the event probabilities of the multinomial model. For each draw from the multinomial, an OTU was accepted to be present 
if at least two reads were binned to it. The number of OTUs was calculated from 5000 draws. The sample size was varied starting from the number of all sequences $(531,442)$ to lower numbers at a decrement of 10,000 , and the mean number of OTUs over 5000 draws was calculated for each sampling. Quantiles for OTU numbers in each sample of 5000 draws was calculated for probabilities 0.95 and 0.05 .

To estimate the sampling distribution of numbers of assigned taxa in synthetic reads data, the results of taxonomic assignment were bootstrapped 1000 times for each taxonomic level and employed database/assignment algorithm combination at that level. The proportion of present vs. absent taxa was calculated for each bootstrap sampling iteration.

To compare absolute and relative numbers of assigned taxa between databases in 454 and Illumina sequencing data sets, paired two-way Wilcoxon signed rank test was performed. All analyses were performed in R software $\mathrm{v}$. 3.1.1 [51].

\section{Availability of supporting data}

HITdb is available in GitHub at https://github.com/microbiome/HITdb.git.

For direct download, use https://github.com/microbiome/HITdb/archive/master.zip.

The contained README file provides instructions and other information.

\section{Additional files}

Additional file 1: Phylogenetic trees. Package containing Newick and figure files of bacterial and archaeal phylogenies in HITdb. (PDF $22521 \mathrm{~kb}$ )

Additional file 2: Computational rarefaction. The figure shows the mean number of OTUs calculated from 5000 draws at different sample sizes from multinomial distribution. The dashed lines indicate the 0.95 and 0.05 quantiles. The horizontal red dotted line marks the number of all found clusters in the original data. (PDF $71 \mathrm{~kb}$ )

Additional file 3: Rarefaction based on known taxa. The figure shows the numbers of taxa calculated from samples of sequence data used for constructing the HITdb. Boxplots showing the numbers of found species (A) and genera (B) at two sample sizes (about $90 \%$ and $80 \%$ of sequences, $n=9$ and $n=10$, respectively). The horizontal red dashed line shows the number of OTUs in all sequences (100\% of sequences). (PDF $52 \mathrm{~kb}$ )

Additional file 4: Synthetic reads. A table listing the V1-V3 and V4-V6 $16 \mathrm{~S}$ sequences from known human intestinal bacterial species used in the study. (XLS $1406 \mathrm{~kb}$ )

Additional file 5: Assignment of synthetic reads. The figure shows the relative numbers of assigned taxa for synthetic reads using Silva, Greengenes and HITdb databases, and RDP, Uclust and Mothur algorithms. (PDF $5 \mathrm{~kb}$ )

Additional file 6: Proportion of missing taxonomic assignments. The figure shows taxonomic assignments missing from Phylum level downwards in Greengenes and HITdb. The data is from two sets of biological samples sequenced either with 454 or Illumina MiSeq. (PDF 91 kb)

Additional file 7: SRA ID codes of HMP samples. A Table listing the Sequence Read Archive accession codes of the Human Metaproteome Project samples used in the study. (XLS $30 \mathrm{~kb}$ )
Additional file 8: Comparison of memory and time usage. The figure shows memory and time usage in HITdb and Greengenes databases. The measurement was done using $937 \mathrm{~V} 4-\mathrm{V} 6$ sequences from human intestinal bacterial species. (PDF $4 \mathrm{~kb}$ )

\section{Competing interests}

The authors declare that they have no competing interests.

\section{Authors' contributions}

$J R$, JS, LL and WMdV conceived the study. JR analysed the data and drafted the manuscript. JS, LL and WMdV critically revised the manuscript. All authors read and approved the final manuscript.

\section{Acknowledgements}

The study was financially supported by the Academy of Finland and the European Research Council (grant 250172, MicrobesInside). LL was supported by the Academy of Finland (grant 256950).

We would like to thank Dr. Anne Salonen for providing sequencing data.

\section{Author details}

${ }^{1}$ Department of Veterinary Biosciences, University of Helsinki, Helsinki, Finland. 'Laboratory of Microbiology, Wageningen University, Wageningen, the Netherlands. ${ }^{3}$ Department of Bacteriology and Immunology, University of Helsinki, Helsinki, Finland.

Received: 11 March 2015 Accepted: 1 December 2015

Published online: 12 December 2015

\section{References}

1. Human Microbiome Project Consortium. Structure, function and diversity of the healthy human microbiome. Nature. 2012;486(7402):207-14.

2. Cheng J, Palva AM, de Vos WM, Satokari R. Contribution of the intestinal microbiota to human health: from birth to 100 years of age. Curr Top Microbiol Immunol. 2013;358:323-46.

3. Guarner F, Malagelada JR. Gut flora in health and disease. Lancet. 2003; 361(9356):512-9.

4. O'Hara AM, Shanahan F. The gut flora as a forgotten organ. EMBO Rep. 2006;7(7):688-93.

5. Kau AL, Ahern PP, Griffin NW, Goodman AL, Gordon J. Human nutrition, the gut microbiome and the immune system. Nature. 2011;474(7351):327-36.

6. Hart AL, Lammers K, Brigidi P, Vitali B, Rizzello F, Gionchetti P, et al. Modulation of human dendritic cell phenotype and function by probiotic bacteria. Gut. 2004:53(11):1602-9.

7. Kalliomaki M, Collado MC, Salminen S, Isolauri E. Early differences in fecal microbiota composition in children may predict overweight. Am J Clin Nutr. 2008;87(3):534-8.

8. Le Chatelier E, Nielsen T, Qin J, Prifti E, Hildebrand F, Falony G, et al. Richness of human gut microbiome correlates with metabolic markers. Nature. 2013;500(7464):541-6.

9. Caporaso JG, Lauber CL, Walters WA, Berg-Lyons D, Huntley J, Fierer N, et al. Ultra-high-throughput microbial community analysis on the Illumina HiSeq and MiSeq platforms. ISME J. 2012;6(8):1621-4.

10. Hamady M, Knight R. Microbial community profiling for human microbiome projects: Tools, techniques, and challenges. Genome Res. 2009;19(7):1141-52.

11. Mosher JJ, Bernberg EL, Shevchenko O, Kan J, Kaplan LA. Efficacy of a 3rd generation high-throughput sequencing platform for analyses of 16S rRNA genes from environmental samples. J Microbiol Methods. 2013;95(2):175-81.

12. Binladen J, Gilbert MT, Bollback JP, Panitz F, Bendixen C, Nielsen R, et al. The use of coded PCR primers enables high-throughput sequencing of multiple homolog amplification products by 454 parallel sequencing. PLoS One. 2007:2(2):e197.

13. Quast C, Pruesse E, Yilmaz P, Gerken J, Schweer T, Yarza P, et al. The SILVA ribosomal RNA gene database project: improved data processing and web-based tools. Nucleic Acids Res. 2013;41(Database issue):D590-6.

14. Yilmaz P, Parfrey LW, Yarza P, Gerken J, Pruesse E, Quast C, et al. The SILVA and "All-species Living Tree Project (LTP)" taxonomic frameworks. Nucleic Acids Res. 2014:42(Database issue):D643-8.

15. DeSantis TZ, Hugenholtz P, Larsen N, Rojas M, Brodie EL, Keller K, et al. Greengenes, a chimera-checked 16S rRNA gene database and workbench compatible with ARB. Appl Environ Microbiol. 2006;72(7):5069-72. 
16. Cole JR, Wang Q, Fish JA, Chai B, McGarrell DM, Sun Y, et al. Ribosomal Database Project: data and tools for high throughput rRNA analysis. Nucleic Acids Res. 2014;42(Database issue):D633-42.

17. Janda JM, Abbott SL. 16S rRNA gene sequencing for bacterial identification in the diagnostic laboratory: pluses, perils, and pitfalls. J Clin Microbiol. 2007; 45(9):2761-4.

18. Mende DR, Sunagawa S, Zeller G, Bork P. Accurate and universal delineation of prokaryotic species. Nat Methods. 2013;10(9):881-4.

19. Edgar RC. UPARSE: highly accurate OTU sequences from microbial amplicon reads. Nat Methods. 2013;10(10):996-8.

20. Liu Z, DeSantis TZ, Andersen GL, Knight R. Accurate taxonomy assignments from $16 \mathrm{~S}$ rRNA sequences produced by highly parallel pyrosequencers. Nucleic Acids Res. 2008;36(18):e120.

21. Huse SM, Dethlefsen L, Huber JA, Mark Welch D, Relman DA, Sogin ML. Exploring microbial diversity and taxonomy using SSU rRNA hypervariable tag sequencing. PLoS Genet. 2008;4(11):e1000255.

22. Bowen De Leon K, Ramsay BD, Fields MW. Quality-score refinement of SSU rRNA gene pyrosequencing differs across gene region for environmental samples. Microb Ecol. 2012;64(2):499-508.

23. Werner JJ, Koren O, Hugenholtz P, DeSantis TZ, Walters WA, Caporaso JG, et al. Impact of training sets on classification of high-throughput bacterial 16s rRNA gene surveys. ISME J. 2012;6(1):94-103.

24. Lahti L, Salojarvi J, Salonen A, Scheffer M, de Vos WM. Tipping elements in the human intestinal ecosystem. Nat Commun. 2014;5:4344.

25. Qin J, Li R, Raes J, Arumugam M, Burgdorf KS, Manichanh C, et al. A human gut microbial gene catalogue established by metagenomic sequencing. Nature. 2010;464(7285):59-65.

26. Eckburg PB, Bik EM, Bernstein CN, Purdom E, Dethlefsen L, Sargent M, et al. Diversity of the human intestinal microbial flora. Science. 2005; 308(5728):1635-8

27. Rajilic-Stojanovic M, de Vos WM. The first 1000 cultured species of the human gastrointestinal microbiota. FEMS Microbiol Rev. 2014;38(5):996-1047.

28. Schloss PD, Gevers D, Westcott SL. Reducing the effects of PCR amplification and sequencing artifacts on 165 rRNA-based studies. PLoS One. 2011;6(12):e27310.

29. Kunin $V$, Engelbrektson A, Ochman $H$, Hugenholtz P. Wrinkles in the rare biosphere: pyrosequencing errors can lead to artificial inflation of diversity estimates. Environ Microbiol. 2010;12(1):118-23.

30. Koeppel AF, Wu M. Surprisingly extensive mixed phylogenetic and ecological signals among bacterial Operational Taxonomic Units. Nucleic Acids Res. 2013;41(10):5175-88.

31. Schloss PD, Westcott SL. Assessing and improving methods used in operational taxonomic unit-based approaches for 16S rRNA gene sequence analysis. Appl Environ Microbiol. 2011;77(10):3219-26.

32. Drancourt M, Bollet C, Carlioz A, Martelin R, Gayral JP, Raoult D. 165 ribosomal DNA sequence analysis of a large collection of environmental and clinical unidentifiable bacterial isolates. J Clin Microbiol. 2000;38(10): 3623-30.

33. Schloss PD, Handelsman J. Toward a census of bacteria in soil. PLoS Comput Biol. 2006;2(7):e92.

34. Schloss PD, Westcott SL, Ryabin T, Hall JR, Hartmann M, Hollister EB, et al. Introducing mothur: open-source, platform-independent, community-supported software for describing and comparing microbial communities. Appl Environ Microbiol. 2009;75(23):7537-41.

35. Li J, Jia H, Cai X, Zhong H, Feng Q, Sunagawa S, et al. An integrated catalog of reference genes in the human gut microbiome. Nat Biotechnol. 2014; 32(8):834-41.

36. Zoetendal EG, Rajilic-Stojanovic M, de Vos WM. High-throughput diversity and functionality analysis of the gastrointestinal tract microbiota. Gut. 2008; 57(11):1605-15.

37. Wang Q, Garrity GM, Tiedje JM, Cole JR. Naive Bayesian classifier for rapid assignment of rRNA sequences into the new bacterial taxonomy. Appl Environ Microbiol. 2007;73(16):5261-7.

38. Di Rienzi SC, Sharon I, Wrighton KC, Koren O, Hug LA, Thomas BC, et al. The human gut and groundwater harbor non-photosynthetic bacteria belonging to a new candidate phylum sibling to Cyanobacteria. Elife. 2013;2:e01102.

39. Soro V, Dutton LC, Sprague SV, Nobbs AH, Ireland AJ, Sandy JR, et al. Axenic culture of a candidate division TM7 bacterium from the human oral cavity and biofilm interactions with other oral bacteria. Appl Environ Microbiol. 2014;80(20):6480-9.
40. Borrel G, Harris HM, Parisot N, Gaci N, Tottey W, Mihajlovski A, et al. Genome Sequence of "Candidatus Methanomassiliicoccus intestinalis" Issoire-Mx1, a Third Thermoplasmatales-Related Methanogenic Archaeon from Human Feces. Genome Announc. 2013, 1(4):10.1128/genomeA.00453-13.

41. Edgar RC, Haas BJ, Clemente JC, Quince C, Knight R. UCHIME improves sensitivity and speed of chimera detection. Bioinformatics. 2011;27(16): 2194-200.

42. Caporaso JG, Kuczynski J, Stombaugh J, Bittinger K, Bushman FD, Costello EK, et al. QIIME allows analysis of high-throughput community sequencing data. Nat Methods. 2010;7(5):335-6.

43. Edgar RC. MUSCLE: multiple sequence alignment with high accuracy and high throughput. Nucleic Acids Res. 2004;32(5):1792-7.

44. Price MN, Dehal PS, Arkin AP. FastTree: computing large minimum evolution trees with profiles instead of a distance matrix. Mol Biol Evol. 2009;26(7): 1641-50.

45. Muyzer G, de Waal EC, Uitterlinden AG. Profiling of complex microbial populations by denaturing gradient gel electrophoresis analysis of polymerase chain reaction-amplified genes coding for $16 \mathrm{~S}$ rRNA. Appl Environ Microbiol. 1993;59(3):695-700.

46. Amann Rl, Ludwig W, Schleifer KH. Phylogenetic identification and in situ detection of individual microbial cells without cultivation. Microbiol Rev. 1995;59(1):143-69.

47. Kumpu M, Kekkonen RA, Kautiainen H, Jarvenpaa S, Kristo A, Huovinen P, et al. Milk containing probiotic Lactobacillus rhamnosus GG and respiratory illness in children: a randomized, double-blind, placebo-controlled trial. Eur J Clin Nutr. 2012;66(9):1020-3.

48. Kuitunen M, Kukkonen $K$, Juntunen-Backman K, Korpela R, Poussa T, Tuure T, et al. Probiotics prevent lgE-associated allergy until age 5 years in cesareandelivered children but not in the total cohort. J Allergy Clin Immunol. 2009; 123(2):335-41.

49. Edwards U, Rogall T, Blocker $H$, Emde M, Bottger EC. Isolation and direct complete nucleotide determination of entire genes. Characterization of a gene coding for 165 ribosomal RNA. Nucleic Acids Res. 1989;17(19):7843-53.

50. Pages H, Aboyoun P, Gentleman R, DebRoy S. Biostrings: String objects representing biological sequences, and matching algorithms. 2014. p. 2.32.1.

51. R Development Core Team. R: A language and environment for statistical computing. 2014. p. 3.1.1.

52. Edgar RC. Search and clustering orders of magnitude faster than BLAST. Bioinformatics. 2010;26(19):2460-1.

\section{Submit your next manuscript to BioMed Central and we will help you at every step:}

- We accept pre-submission inquiries

- Our selector tool helps you to find the most relevant journal

- We provide round the clock customer support

- Convenient online submission

- Thorough peer review

- Inclusion in PubMed and all major indexing services

- Maximum visibility for your research

Submit your manuscript at www.biomedcentral.com/submit 